\title{
EL ESPACIO PÚBLICO: CONSTRUCCIÓN Y APROPIACIÓN DESDE LA INFORMALIDAD
}

\author{
Jeison Andres Hincapie Rodriguez \\ Universidad Nacional de Colombia \\ Email: jeahincapiero@unal.edu.co, jeian91@gmail.com \\ Luz Adriana Castiblanco Martínez \\ Universidad Nacional de Colombia \\ Email: lacastiblancom@gmail.com, luz.castiblanco622@esap.gov.co
}

\section{RESUMEN}

La definición y construcción de los espacios colectivos en los asentamientos informales surgen de la necesidad por parte de la población de generar equipamientos y lugares de encuentro desde donde se gesten relaciones, deseos e imaginarios. En ese sentido, los espacios públicos y las centralidades se han desarrollado de manera atípica y alterna a un proceso de planificación institucional y tradicional, pues si bien existen, no fueron diseñados bajo parámetros del diseño urbano; por el contrario, han sido gestados desde las dinámicas de los ocupantes quienes construyen los espacios colectivos en primera instancia, y luego por uso frecuente promueven una apropiación comunitaria recurriendo a la construcción contante del hábitat y del tejido social en el espacio de los asentamientos informales. A partir de lo anterior, se pretende dar a conocer la importancia que tiene el espacio público para la construcción y mantenimiento de asentamientos, así mismo bajo un proceso de legalización y articulación al perímetro urbano de las ciudades teniendo en cuenta que la informalidad en países de América Latina es un proceso común debido a la presión del mercado inmobiliario y a la baja acción de políticas en torno a la asequibilidad de la vivienda.

Palabras Clave: Construcción social; Hábitat; Espacio Público; Informalidad, Roles, Exclusión

\begin{abstract}
The definition and construction of collective spaces in informal settlements arise from the need by the population of generating facilities and meeting place. So, common areas and inner cities have developed atypical and alternates to institutional planning process so because while there, were not designed under the parameters of urban design; on the contrary, they have been gestated from the dynamics of the occupants who build collective spaces in the first instance, and then by frequent use promote community ownership. From the foregoing, it is intended to show how it is the public space is generated in such settlements considering informality in Latin America is a common process.
\end{abstract}

Key Words: Informal neighborhoods; Social construction; Public space 


\section{INTRODUCCIÓN}

Los estudios desarrollados en torno al acceso, asequibilidad y calidad de la vivienda ocupan un lugar destacado en países de Latinoamérica, debido a que la relación del vaciamiento del campo a la ciudad por los procesos de desarrollo, desplazamientos y otras características propias, han generado ocupaciones ilegales y construcción del hábitat a partir de la informalidad en las grandes urbes, ocasionando que las demandas por un techo no sean subsanadas en su totalidad por los Estados. Ello se ha traducido en inequidades sociales, desigualdad al derecho, goce de la ciudad y desconexión a servicios; por lo tanto, se configuran espacios desde la informalidad con nuevas subjetividades sociales en contextos locales ligados a las transformaciones urbanas, particularmente en las periferias.

Entonces, la definición y construcción de los espacios públicos en los asentamientos informales surgen de la necesidad por parte de la población de generar equipamientos y lugares de encuentro, por lo que los espacios comunes y las centralidades se han desarrollado de manera atípica y alterna a un proceso de planificación institucional, pues si bien existen, no fueron diseñados bajo parámetros del diseño urbano; por el contrario, han sido gestados desde las dinámicas de los ocupantes quienes construyen los espacios colectivos en primera instancia, y luego por uso frecuente promueven una apropiación por parte de la comunidad, lo que propone que los lugares adquieran expresiones ideológicas, culturales y políticas que se mantienen en el tiempo por medio de la interacción y siguiendo a Jane Jacobs la vida en el espacio público posibilita los encuentros entre extraños, que aunque superficiales, pueden ser satisfactorios. (Jacobs, 1961 ) A partir de lo anterior, se pretende ver la importancia del espacio público en este tipo de asentamientos, teniendo en cuenta que la informalidad en países de América Latina es un proceso frecuente y de constante reproducción dada las características de asequibilidad y acceso a la vivienda.

A saber, los habitantes transforman sus espacios urbanos con el uso cotidiano, funcional y simbólico que le otorgan, por lo que este intercambio es en doble vía, ya que el espacio es moldeado pero también lo son las personas que de él hacen uso, en la medida en que la relación ser humano-naturaleza, en este caso el medio urbano-"no formal", son revalidadas. En ese sentido, para la materialización de políticas es fundamental la comprensión del espacio como constructo, pues es allí donde se plantean escenarios que se contraponen a lo formal, pero que por el hecho de no pertenecer a la normatividad deben ser obviados, razón por la cual se homogenizan las relaciones socio-espaciales.

Por esta razón, a través del escrito se pretende destacar la necesidad de explorar las perspectivas de la población con respecto al espacio público en la informalidad. Pues estas composiciones del hábitat forman parte de su cultura y se constituyen desde un saber práctico y de primera mano, que busca resolver los problemas de la vida cotidiana y conformar relaciones sociales más amenas bajo símbolos que rigen la convivencia, en ese sentido se recuerdan factores como la falta de agua, la construcción de la vivienda con materiales poco adecuados, vías de conexión, espacio público y códigos de habitar el lugar. Estas prácticas tienen su propia racionalidad a pesar de que no se encuadren con la visión y lenguaje oficial del diseño urbano, además funcionan bajo principios definidos, con reglas que pueden variar dado el contexto, desde las vivencias cotidianas con el lugar hasta la conformación de la trama la cual es referenciada por (Sepúlveda, 1992) como la organización de elementos espaciales y nodales que se mezclan y que con el espacio geográfico conforman una estructura física del conjunto, la que además es modificada a diario. 

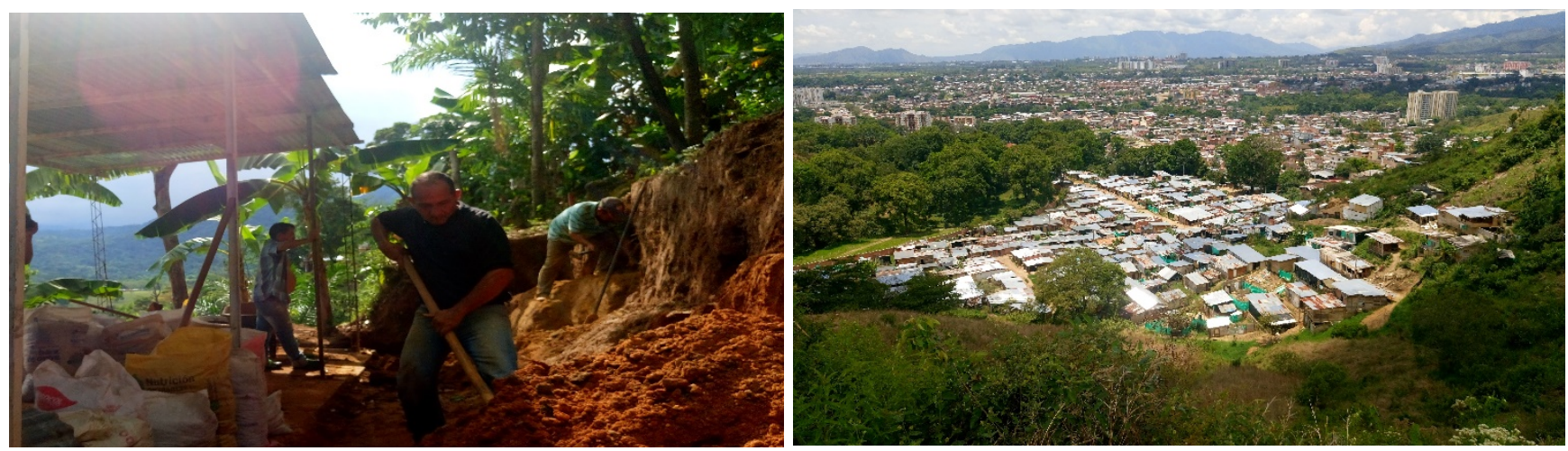

Ilustración 1 Comunidad "adecuando" el suelo de una pendiente con el fin de construir un recinto de reuniones, asentamiento La Nora. Fuente: Elaboración propia.

Ilustración 2. Vista desde el "mirador" del asentamiento Eco-paraíso, al fondo la ciudad formal de Ibagué.

Entonces, la configuración de las redes del habitar se genera a diferentes escalas, en este caso en la calle y en el asentamiento a partir de los recorridos, permanencias e intercambios en torno a un objetivo común en un principio como lo es la construcción y manutención en el tiempo del asentamiento. A diferencia del saber científico y administrativo, que establece un discurso y obliga con base en una lógica pre-establecida, la implementación del entorno producido desde los escritorios y oficinas de planeación. También, respondiendo a lógicas que fueron asumidas de acuerdo a enfoques que los gobiernos aceptaron como una realidad del mundo "subdesarrollado".

Con respecto a la planeación, esta se ha identificado como un enfoque teórico netamente para el análisis de las dinámicas de acceso a la ciudad, crecimiento urbano y asequibilidad a los mínimos de vivienda. Sin embargo, ello se ha venido realizando a partir de un rompimiento en el paradigma, pues en el proceso de construcción de unas políticas pública era menos importante la participación de la ciudadanía y menos aún la construcción del hábitat y de los tejidos sociales a partir de la informalidad urbana, en ese sentido no se puede desligar de la naturaleza, la sociedad que la transforma y el habitante que lo ocupa, pues retomando a (Echeverría, 2009) el hábitat es la trama de relaciones que cohesionan el territorio desde las acciones del habitar en un entorno. De tal manera, se genera una aproximación, somera, espacial y temporal en la comprensión de la vida social contemporánea, lo cual afecta la comprensión de la funcionalidad de las dinámicas en la creación de vínculos emocionales con el espacio público, en una forma de inclusión simbólica que re-significa la dignidad espacial y con ello el derecho a la ciudad, en este sentido, es la posibilidad de acceder a un espacio reconocido y disputado a diario.

Siguiendo con lo anterior, estas relaciones también implican la configuración del hábitat, como lo señala (Leff, 2002) es el lugar en el que se construye y se define la territorialidad, la espacialidad de una sociedad, donde se constituyen los sujetos sociales que diseñan el espacio geográfico, apropiándoselo, habitándolo con sus significaciones. Por tal motivo, el documento pretende generar una postura articulada entre el análisis de la importancia del espacio público desde la informalidad, políticas de mejoramiento barrial y recuperación de espacio público, acudiendo a una visión desde el asentamiento informal. Con ello demostrar la importancia de las construcciones del espacio, el cual no se limita a condiciones físicas, sino está cargado de subjetividades y vivencias ya que se han afectado las dimensiones sociales y el desarrollo urbano tras la implantación de programas que no recogen la información de la comunidad o que la omiten.

En tal sentido, se presenta como hipótesis que las políticas inciden directamente en condiciones de acceso y aceptación de los espacios públicos y equipamientos, lo que conlleva a la imposición de relaciones de dominación, poder y rechazo, generando así, cambios en la construcción social del espacio urbano y hábitat. Pues los órdenes sociales y simbólicos responden a significaciones y funcionalidades que rodean el contexto urbano, así como lo hacen las variables políticas, económicas e institucionales que terminan configurando las tramas del hábitat y de la vida en sociedad.

Se estructuró así una ruta en la que se exponen el espacio público como un constructo social configurado por las relaciones propias de la autogestión; en segundo lugar se realiza una aproximación a la articulación entre las políticas y programas de recuperación del espacio público y mejoramiento barrial a partir de la relación con los seres humanos, haciendo referencia al conflicto por las territorialidades y la construcción del hábitat; en tercer 
lugar se hará referencia a una ejercicio de campo en asentamientos informales de Villavicencio e lbagué (Colombia), en la que se puede observar las construcciones sociales con referente a la continuidad y tejido del espacio público en el territorio. Finalmente se plasman unas conclusiones

Se trataría, entonces, de desarrollar una perspectiva de planeación urbana desde la geografía y el hábitat que evidencie las prácticas cotidianas de los individuos y sus dimensiones simbólicas con respecto a los lugares, el barrio, el conjunto, etc. Es de agregar que se analizan las representaciones que los habitantes de dos asentamientos informales han dado a sus dinámicas de construcción del entorno a partir de la informalidad e ilegalidad en la tenencia de la tierra, que en ocasiones se fortalecen los lazos debido al objetivo en común hacia un proceso de legalización.

\section{Reconocimiento del espacio público como un hecho social}

Los planificadores del mundo industrializado han realizado, a modo de recetario, "bolsas" de programas y políticas que conducen por el camino del bienestar a países que aún no logran las metas planteadas para alcanzar el estado anhelado de desarrollo. En este sentido, se crearon, difundieron y pusieron en agenda algunos enfoques, los cuales luego de ser aceptados por los organismos multilaterales e influenciadas por las lógicas económicas frente al cambio de modelo de provisión de la vivienda, se convirtieron en políticas públicas del hábitat y de vivienda medidas con el miso racero, y en ocasiones dejando por fuera posturas de la ciudadanía receptora de las mismas.

Estos enfoques han tenido la pretensión de comprender la estructura social, económica y cultural en que transcurre la vida de los habitantes y el sentido propio que otorgan a sus prácticas y percepciones urbanas. Ello ha incidido de manera negativa pues las visiones no son estáticas, cambian conforme a las coyunturas del momento y de la morfología del lugar, en este sentido se cuenta por ejemplo, que para la planificación urbana, se pasó de la arquitectura moderna a perspectivas sociológicas, de acuerdo con (Duque, 2015) sin que por ello se hayan atendido las necesidades de las personas, por ejemplo en asentamientos de origen informal, donde se consideran practicas sin forma y estética.
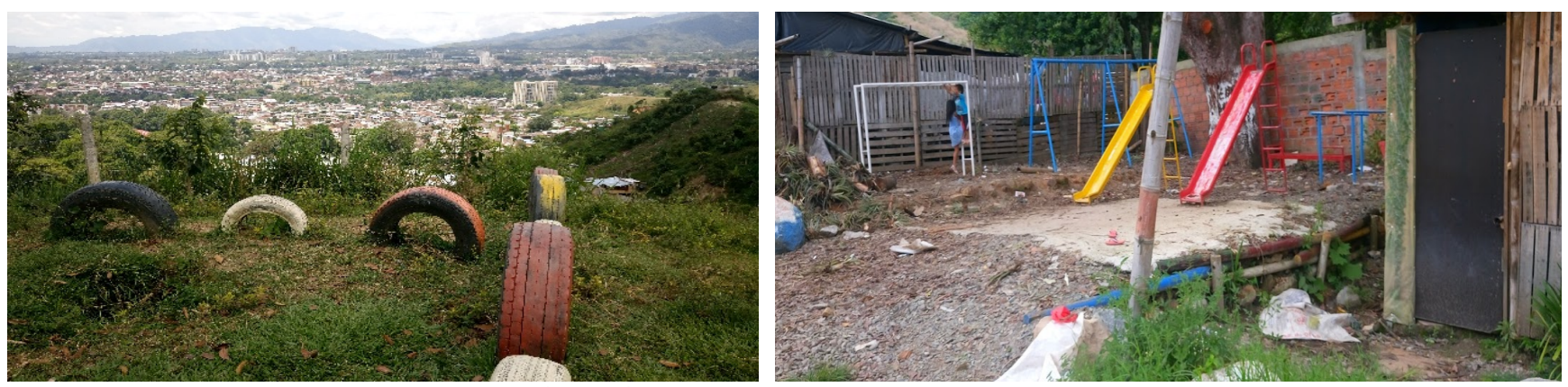

Ilustración 3 Asentamiento Informal Ecoparaiso (Ibagué) En la imagen de la derecha se observa un espacio de encuentro para la realización de asambleas, además de constituirse en un "mirador".

llustración 4. En la imagen de la derecha, se observa un parque infantil construido por los habitantes a partir de la autogestión. Fuente: Elaboración propia

En ese sentido, a partir del viraje de los enfoques del urbanismo se han desarticulado a las personas de los procesos, obviando las relaciones de estas con el espacio urbano, pues en él se desarrollan dinámicas económicas, espaciales y sociales, las cuales promueven efectos específicos sobre la estructura de la ciudad. Así, la implementación de programas de construcción de vivienda en masa y legalización plantea un cambio en las dinámicas y relaciones que los "beneficiarios" tienen con el territorio, por lo que no se genera la normal conectividad, relaciones sociales e identidades territoriales a partir de las subjetividades, necesidades y trayectorias que se reproducen.

Ahora bien, el proceso planificador le ha dado peso a discursos tecnocráticos y de las dinámicas económicas en las que se encuadran las ciudades, lo que ha llevado a que la competitividad por lograr ofertas espaciales aceptables para el turismo e inversión, modifiquen de manera funcional los espacios públicos, equipamientos y calles que deben ser ordenadas y representativas del desarrollo. Por lo que los individuos pueden percibir que 
no tienen derecho de acceso a la ciudad y a los servicios que esta ofrece y es en este escenario en el que exige modificaciones a la planificación existente con el fin de que se cumplan las expectativas que se han construido por medio de la cotidianidad, a partir de la gestión de espacios en el que se construyen escenarios locales y calidad de vida, por lo que el hábitat informal se debe trasformar a partir de este escenario. En ese sentido la naturaleza de las políticas públicas y la apropiación del espacio urbano cotidiano y popular, deben ser tenidas en cuenta, a la hora de producción, mejoramiento, difusión y aceptación por parte de la ciudad tradicionalmente construida.

Este apartado pretende identificar cómo el Estado reproduce una mirada hegemónica sobre la manera en que se entiende una determinada problemática a escala urbana, y qué imaginarios y constructos sociales se están generando a partir de la implantación del urbanismo tradicional y que plantea el mejoramiento de barrios. En ese sentido, (Soja, 2009) afirma que el espacio es un producto social donde se gestan las justicas e injusticias de acceso, por lo que el espacio tiende a modelar las relaciones en las pueden permear las injusticias. Así, las desigualdades, no se reproducen por sí mismas, sino dependen de las políticas públicas que se adoptan para el momento y de la manera en que son recibidas por la población.

También, siguiendo a (Soja, 2009) la discriminación de la localización de viviendas y espacio público, ha creado sesgos e impuesto cargas a una determinada población debido a la ubicación geográfica o el espacio ocupado en la ciudad, evidenciado en la injusticia y en las estructuras espaciales que se localizan. Ello se observan en la vida cotidiana y por lo que depende de las políticas y programas construir escenarios integradores de la forma como actúa el Estado.

En tal sentido, (Sabatini y Brain, 2012) señalan que las desigualdades sociales no son vistas como estructurales o problemas político-administrativos, sino más bien se ve que surgen a partir de decisiones que los individuos han hecho, sin tener en cuenta las limitaciones que pudiesen encontrar, ello se relaciona con la informalidad como salida al déficit de vivienda, por lo que la población pretende la distribución en el espacio social a partir de valorar los recursos y las oportunidades de quienes lo utilizan y construyen desde la cotidianidad y de los usos que se le pueden dar a un mismo espacio, por ejemplo la calle y zonas donde se da la vida cotidiana, a diferencia de la visión estatal, la cual postula la relación jerárquica que se ha establecido entre el Estado y las "beneficiarios" de los programas y políticas, con el fracaso de las mismas, su aceptación o las actitudes de resistencia ante los cambios.

Así y como postulado del documento, mientras que se destaca desde la informalidad la ocupación o loteo, se generan los cambios en la construcción del hábitat y la morfología de los asentamientos, los cuales han estado mediados por la articulación con el entorno físico inmediato, (Yory, 2015) ya que este imprime límites, materiales, formas en que deben construirse las viviendas, espacios comunes y de tránsito lo cual responde a las trasformaciones tanto económicas como políticas urbanas, ya que los componentes del sistema no son independientes y que para el caso del hábitat no se puede desligar de la naturaleza, la sociedad que la transforma y el habitante que lo ocupa, pues retomando a (Echeverría, 2009) El hábitat es la trama de relaciones que cohesionan el territorio desde las acciones del habitar en un entorno.

Con todo ello, el espacio se convierte en un elemento clave en el análisis de los roles, de las territorialidades y de la autogestión para mejorar las condiciones de habitabilidad y encuentro, con lo cual se crean nuevas percepciones sociales. Es así como el concepto de espacio pasa a adquirir una significación muy amplia representando la suma del contexto geográfico y de la trama cultural. De acuerdo a lo anterior, (Sabaté A, 1995) afirma que la redefinición de conceptos se gesta a través de sectores informales, pues estos definen los espacios públicos por medio de los usos y costumbres a partir de sentidos de pertenencia, poder e integración. Así pues, en los asentamientos informales las representaciones subjetivas del espacio, imaginarios y usos se pueden considerar como una herramienta de patrones culturales presentes para un futuro imaginado a intervenir.

\section{Espacio público desde los instrumentos y programas de mejoramiento}

La actuación de los entes administrativos se materializa por medio de las políticas públicas, por lo cual se convalida una visión de cómo las instituciones y la sociedad deben entender e intervenir sobre los problemas que son priorizados. Es decir las situaciones problemáticas que el Estado define como relevantes, en este punto se hace necesario aclarar que el mejoramiento barrial y procesos de legalización, que además de escasos y 
fraccionados, tienden a reducir las prácticas cotidianas y funcionales asociadas con los espacios públicos, que representa las distintas formas y necesidades de cómo los habitantes se relacionan con esos lugares, deconstruyendo un vínculo de apropiación y resistencia que conlleva a la transformación en la morfología de comunidades de los territorios a intervenir, evidenciado en los procesos inconclusos de legalización.

Así, y como producto del trabajo en algunos barrios de tipo informal en Colombia, es que las políticas públicas no pueden ser elaboradas a partir de datos netamente cuantitativos y de la visión reduccionista del diseño urbano tradicional; por el contrario deben ser el resultado de representaciones, construcciones y concepciones, en este caso de las formas en cómo se vive, transita y disfruta el espacio público, y además dependen de un contexto y constructos sociales previamente establecidos y constantemente re-legitimados, así, Velásquez (2009), conceptualiza la política pública como:

Un proceso integrador de decisiones, acciones, inacciones, acuerdos e instrumentos, adelantado por autoridades públicas con la participación eventual de los particulares, y encaminado a solucionar o prevenir una situación definida como problemática. La política pública hace parte de un ambiente determinado del cual se nutre y al cual pretende modificar o mantener (Velásquez, 2009, pág. 156)

A pesar de lo anotado anteriormente, se han masificado posturas en las que se implantan visiones en las que el espacio público tiene un papel exclusivo de recreación y esparcimiento desde lo formal, sin tener en cuenta que se pueden dar relaciones sociales y vínculos de apego en contravía de poder de quienes lo ejecutan, en este caso desde las ciudades y municipios por instrucción del Ministerio de vivienda, ciudad y territorio. Al respecto, Majone ve en las políticas públicas una construcción de palabras, entendidas como "el arte de la persuasión, el estudio de todas las formas de hacer cosas con palabras" (Majone, 1997, pág. 23) es decir, que se pueden responder a lógicas en donde se "formaliza" el espacio público con el fin de contribuir a la venta de servicios y las city marketing.

Cabe profundizar en este último término, que se genera por medio de la competitividad en el mercado global y con ello concebir inclusión y equidad; lo anterior, tiene una relación directa con la disposición del espacio público, ya que si no se ataca la informalidad e intervienen los espacios que han sido desarrollado en torno a esta, pocas oportunidades tiene una ciudad "marketing" pues su imagen pauperizada no venderá en la misma proporción en la que se disminuya la inequidad social, se limpien las calles y se promueva todo un discurso de pertenencia, a pesar de que sea de percepción y en ocasiones no real. Ello se puede observar con la inclusión y equidad territorial, la que se genera, de acuerdo con (Duque, 2015) en una forma de inclusión simbólica que re-significa la dignidad espacial y con ello el derecho a la ciudad, y en este caso al espacio público.

Ante lo anteriormente expuesto, (Fischer, 2003) establece que las políticas públicas son construcciones discursivas, en las que se congregan discursos dominantes. Sin embargo, el espacio es permeado por quienes lo transitan y se construyen desde la informalidad, por lo que las políticas e intervenciones en pro de las mejoras de las condiciones de vida deben tener en cuenta no solo las historias, sino también las no-historias que hacen referencia a las críticas de políticas públicas, que han sido débiles en el escenario de discusión con lo local, y que por ende, no tienen en cuenta las subjetividades multiescalares espaciales, mucho menos el hábitat que se convierte en un elemento clave en la disposición de los roles, de las relaciones económicas y percepciones sociales de acuerdo a la diferenciación de ubicación, patrón de conformación y participación comunitaria en la construcción del asentamiento. Entonces el concepto de hábitat pasa a adquirir una significación muy amplia representando la suma del contexto geográfico y cultural, observando las dimensiones sociales, económicas y políticas de la vida tanto individual, como en comunidad, lo cual implica las dimensiones subjetivas del espacio público a partir de lo que (Ortíz, 2004) considera como el asentamiento y percepción del individuo.

Por consiguiente, es importante describir la manera en que se generan los discursos hegemónicos por medio de la exploración de las posturas de algunos actores que intervienen en las fases de la legalización y mejoramiento barrial, ya que los argumentos de la política no cubren realmente a los ciudadanos, pues tienen fines económicos sobre los espacios intervenidos. Como se pudo constatar en campo, la organización del espacio urbano responde a relaciones de poder y favores burocráticos, caso contrario al espacio público el cual surge como una manifestación social que se conecta con las visiones de los actores y que los agentes (para los casos estudiados, alcaldías municipales) deben respetar y adecuar conforme a lo construido previamente. 
En efecto, se destaca que los fenómenos de desigualdad y discriminación en la política pública pueden llegar a desarrollar procesos de exclusión en los discursos de la cotidianidad, cuando han sido llevados a cabo programas que no responden a la realidad del territorio. De esta manera, se observa que la política pública de vivienda no se encuentra dentro de las transformadoras de la realidad, pues siguen asignando roles a la sociedad lo que promueve la continuidad en la segregación espacial.

También, es importante mencionar que los espacios no son modificados necesariamente y dependen de otras variables, tales como economías locales, costumbres y frecuencia de uso, lo cual no supone que sin equipamientos y espacios se regresará a la esfera de lo privado, aunque no es de desconocer que dificultará la presencia en las demás esferas en la vida social y más aún cuando no se reconoce la importancia del espacio público para la informalidad, como parques, calles, canchas de juego, etc., que promueven el empoderamiento y vínculo entre la comunidad, lo cual es vital para la planificación sobre los procesos de densificación, transformación de los usos del suelo y la complejización de la estructura social en las ciudades latinoamericanas que son contenedoras de la informalidad.

De otra parte, algunas corrientes de la geografía hacen énfasis en las formas de los paisajes y en las relaciones sociales que se construyen y reproducen, de tal forma los paisajes son entendidos ya no como algo per sé, sino que refleja las relaciones de poder y las formas dominantes y hegemónicas de ver el territorio, por medio de la apropiación y construcción del hábitat. En ese sentido, la relación que surge entre la informalidad y espacio público se origina a partir de que este último no es neutro. Luego, es necesario incorporar las diferencias sociales en el análisis espacial y territorial, porque ellas permiten entender las claves de la organización de la sociedad y la posibilidad de acceso al espacio utilizado como medio de control social y político, pues entre otras tiende a aumentar la gobernabilidad (Yory, 2015). Con el fin de evitar lo que (Delgado, 2003)señala como la desigualdad se especializa, generando la lucha política por el espacio y las espacialidades alternativas que incluyen la esfera del hogar, el trabajo, la recreación y la vida comunitaria, como se pudo evidenciar a lo largo de la investigación.

Es así que se hace fundamental entender las representaciones sociales sobre el espacio público y sus roles socialmente asignados, para una transformación cultural por medio de la producción de discursos alternativos, así como también los paradigmas establecidos que se conservan de las personas que engrosan la informalidad. Por lo que el análisis de los roles asignados a las esferas pública y privada, permite identificar que desde la formalidad se siguen reproduciendo los mismos estereotipos de relacionar la calle como un simple espacio de tránsito, el parque como de esparcimiento y juego, acentuando un sesgo que existe en la investigación desde los estudios sobre el espacio y planificación urbana, quizás debido a la preponderancia que presenta el modelo económico. Sin embargo, no en todos los casos se puede generalizar este hecho, pues como se evidenció en estos espacios se tejen redes sociales, democratización de las decisiones en pro del desarrollo urbanístico de los asentamientos, encuentro y apropiación del espacio que articulan las acciones comunitarias ejercidas por los actores.

De tal manera, (Raffestin, 1993) entiende las relaciones con el territorio como las manifestaciones espaciales de poder con la presencia de actores, acciones e instituciones, las que se convierten en símbolos de la construcción, de manera que el asentamiento se genera a partir del espacio público y de las acciones que las personas construyen en torno a este. Por tanto, en este apartado se realizó una observación acerca de la importancia de relacionar identidades culturales, las formas de apropiación y las normatividad lo que ha generado sobre los habitantes roles, así como una preocupación por el acceso al espacio público para la construcción de identidades. Finalmente, es necesaria la construcción articulada de una política de vivienda, pues el espacio urbano no se aparta de una configuración social de este, por lo que incluye espacios de esparcimiento, redes viales, de infraestructura y de servicios públicos, entre otros.

Entendiendo que las dinámicas socioeconómicas condicionan la vida de la población residente y nueva al interior del territorio, por lo que deben conocerse con el fin de evitar segregaciones espaciales, así mismo, en la dotación de espacio público y equipamientos, como lo señalan (Aguliar y Yepes, 2013) es importante alinear las inversiones en vivienda con la provisión de equipamientos, servicios públicos y transporte y espacio desde la comunidad que los ha creado y conservado, como mecanismos para combatir los efectos de la segregación. Por lo tanto, la planificación del territorio debe responder a la reorientación de las relaciones entre ser humano y espacio urbano, permitiendo una armonía entre la construcción del mismo y de las lógicas de integración con las cuales se le ha dado forma. 
De acuerdo a esta postura, se desarrolla un espacio público posteriormente segmentado, lo cual modificaría los imaginarios, estigmas y el diario vivir. Esto potenciado por la incapacidad de la administración pública a la hora de resolver los problemas de conflicto por comprender dichas relaciones, recordando a (Augé, 1996) la simbolización del espacio es un proceso que remite al establecimiento de límites, proceso íntimamente ligado a la identidad y a la diferencia, apartando a quienes constituyen parte de la población, es decir afectando los lazos del hábitat, entendiendo este como un conjunto de dinámicas e interacciones entre elementos de distintas dimensiones económicas, físicas, culturales, naturales y socio-políticas que configuran la vida en la ciudad.

Finalmente la relación entre el crecimiento económico sostenido y la generación de mejores condiciones de vida en las áreas urbanas, involucra mecanismos de gestión urbana y de hábitat, así como, de acuerdo con (Giraldo, 2004) el desarrollo humano en los asentamientos precarios los cuales son determinantes para la inversión pública y permiten mitigar la inequidad y la vulnerabilidad urbana y social. En tal sentido, al incorporar aquellas dinámicas que inciden en la planificación, la gestión del riesgo y el ordenamiento territorial, se logran proponer y definir nuevas formas de intervención por parte de la administración pública que comprenda un saber del hábitat, como referencia (Giraldo, 2004) que lleva implícito la articulación de lo físico y lo imaginario desde la casa hasta el entorno del espacio público.

\section{El espacio público desde los asentamientos informales: estudio de caso}

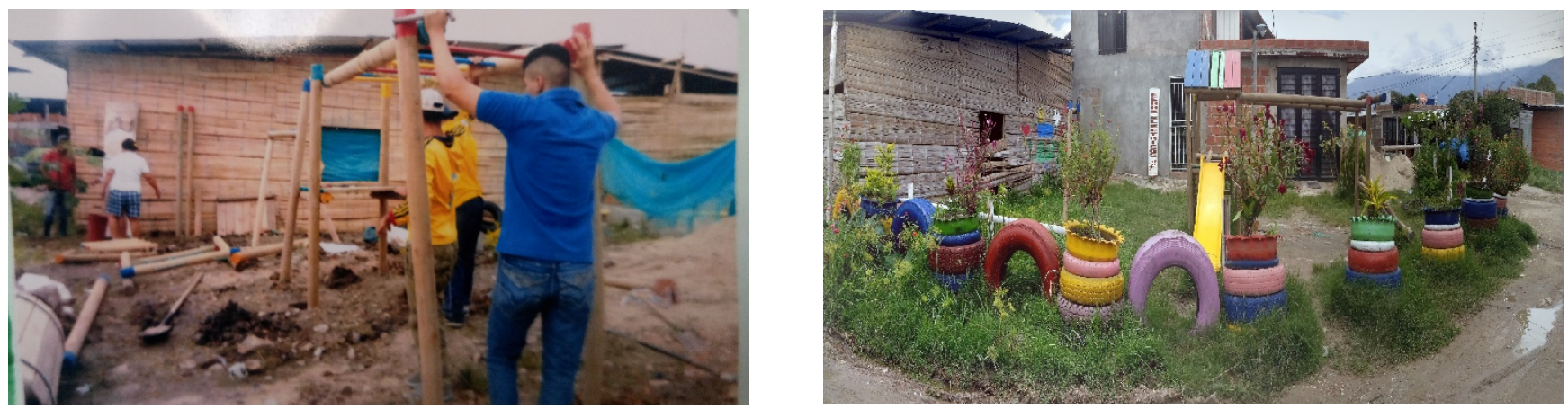

Ilustración 5 Autogestión del parque para niños en el asentamiento informal "Milagro de Dios". Fuente: Registro fotográfico de la comunidad llustración 6. Parque realizado por la comunidad del asentamiento Informal, conformado con materiales reciclables. Fuente: Elaboración propia.

La transformación de las lógicas sociales y económicas de los territorios de cara a la globalización, inciden directamente en la configuración de las ciudades tanto formales, como las de un resultado de procesos informales, lo que se traduce en el aumento en la demanda de suelo urbanizable a través del cual se busca crear y optimizar condiciones de habitabilidad. Como se ha mencionado, el espacio público tiene una estrecha relación con los habitantes de los barrios y más aún de los informales, los cuales tienen ciertas características de uso y apropiación, por ejemplo de recreación, encuentros sociales y culturales; y un punto de referencia e identidad desde la construcción de estos asentamientos.

Así pues, esta disputa por el acceso al suelo genera un choque e impactos a escala local y de ciudad, pues se han gestionado al interior de los asentamiento espacios públicos, que va en contra de lógicas institucionales y que con el transcurrir del tiempo la población que habita en la informalidad se buscará la legalización, provisión de servicios públicos y reconocimiento de estos espacios.

Tabla 1: Caracterización de espacio público en los estudios de caso.

\begin{tabular}{|c|c|c|c|c|}
\hline $\begin{array}{c}\text { Asentamiento } \\
\text { Informal }\end{array}$ & $\begin{array}{c}\text { La Nora } \\
\text { (Villavicencio) }\end{array}$ & $\begin{array}{c}\text { Eco-paraíso } \\
\text { (Ibagué) }\end{array}$ & $\begin{array}{c}\text { Villa Campestre } \\
\text { (Villavicencio) }\end{array}$ & $\begin{array}{c}\text { "Milagro de Dios" } \\
\text { (Ibagué) }\end{array}$ \\
\hline Salón comunal & $\begin{array}{c}\text { Reunión en la cancha } \\
\text { de "tejo" }\end{array}$ & $\begin{array}{c}\text { Dividido en } \\
\text { etapas y reunión }\end{array}$ & $\begin{array}{c}\text { Reunión en las } \\
\text { viviendas de los }\end{array}$ & $\begin{array}{c}\text { Reunión en una } \\
\text { vivienda }\end{array}$ \\
\hline
\end{tabular}




\begin{tabular}{|c|c|c|c|c|}
\hline & & $\begin{array}{c}\text { en casas y zona } \\
\text { verde }\end{array}$ & $\begin{array}{c}\text { líderes del } \\
\text { asentamiento }\end{array}$ & \\
\hline Deportes & Plaza central & $\begin{array}{c}\text { Cancha } \\
\text { autoconstruida }\end{array}$ & $\begin{array}{c}\text { Cancha } \\
\text { multipropósito }\end{array}$ & $\begin{array}{l}\text { Calles y una zona } \\
\text { verde reducida }\end{array}$ \\
\hline $\begin{array}{c}\text { Recreación y } \\
\text { construcción } \\
\text { del tejido social }\end{array}$ & $\begin{array}{c}\text { Un parque de Bolsillo, } \\
\text { por conformado la } \\
\text { comunidad }\end{array}$ & $\begin{array}{l}\text { Un parque para } \\
\text { niños } \\
\text { autoconstruido }\end{array}$ & $\begin{array}{l}\text { Un parque para } \\
\text { niños y jarillon } \\
\text { aprovechado por } \\
\text { la comunidad }\end{array}$ & $\begin{array}{l}\text { Calles y parque } \\
\text { conformado por la } \\
\text { comunidad }\end{array}$ \\
\hline Red vial & $\begin{array}{c}\text { Vía de acceso en } \\
\text { precarias condiciones } \\
\text { y } \\
\text { senderos angostos } \\
\text { en el interior, que } \\
\text { comunican las } \\
\text { viviendas }\end{array}$ & $\begin{array}{l}\text { Vías de acceso } \\
\text { en precarias } \\
\text { condiciones, } \\
\text { caminos } \\
\text { interiores con un } \\
\text { perfil menor. } \\
\text { Dada la } \\
\text { presencia de } \\
\text { Ríos, las vías de } \\
\text { acceso presentan } \\
\text { grave amenaza } \\
\text { de desplome. }\end{array}$ & $\begin{array}{l}\text { Vías de acceso } \\
\text { inexistentes y } \\
\text { desarrollo de vías } \\
\text { internas de forma } \\
\text { precaria, pero } \\
\text { que sirven de } \\
\text { sustento para las } \\
\text { relaciones } \\
\text { humanas. }\end{array}$ & $\begin{array}{c}\text { Vía de acceso en } \\
\text { precarias condiciones } \\
\text { y callejones al interior, } \\
\text { que comunican las } \\
\text { viviendas y permite el } \\
\text { encuentro. }\end{array}$ \\
\hline $\begin{array}{l}\text { Red ambiental } \\
\text { y recreativa }\end{array}$ & $\begin{array}{l}\text { Ubicado al interior de } \\
\text { la red ecológica } \\
\text { principal, causando } \\
\text { un desequilibrio y } \\
\text { daño. }\end{array}$ & $\begin{array}{l}\text { No existe y el } \\
\text { asentamiento se } \\
\text { ubica sobre la } \\
\text { cuenca del rio y } \\
\text { en zona de } \\
\text { deslizamiento. }\end{array}$ & $\begin{array}{l}\text { Las viviendas se } \\
\text { ubican en la cota } \\
\text { del rio, el cual } \\
\text { amenaza con } \\
\text { desbordarse e } \\
\text { inundar el } \\
\text { asentamiento. }\end{array}$ & $\begin{array}{l}\text { Las viviendas se } \\
\text { ubican sobre un } \\
\text { afluente. }\end{array}$ \\
\hline
\end{tabular}

Fuente: Elaboración propia a partir de las visitas a los asentamientos y percepciones a partir de algunos habitantes.

Entonces, los parques, plazas y plazoletas se conforman y construyen desde los imaginarios a su vez como escenarios de encuentro y de vida pública, los cuales difieren en gran medida de los reconocidos por la "otra" ciudad. En ese mismo sentido, los patrones y tendencias en torno a la configuración y dinámicas de uso del territorio, se manifiestan a través de los cambios morfológicos, la definición de tipologías de ciudad, los procesos de urbanización, construcción del tejido social y la disposición de equipamientos públicos. Por lo mencionado, es necesario abordar las dinámicas que han modelado la forma de expansión de ciudades como Villavicencio e Ibagué, con el fin de comprobar la importancia del espacio público el cual complejiza la estructura socia del hábitat, más aún cuando se presenta informalidad en la tenencia de la vivienda, sobre lo cual se enfocó el estudio, comprendiendo las dinámicas en cuanto a la construcción de hábitat y procesos de consolidación del hábitat a partir del uso y apropiación del espacio público.

Entonces, (Pérez y Castellanos, 2013) ve que la definición y construcción de los espacios colectivos en el asentamiento informal, surgen de la necesidad por parte de la población de generar equipamientos y lugares de encuentro, los cuales obedecen a la percepción y organización de las comunidades. En ese sentido, el espacio común y las centralidades, se han desarrollado de manera atípica y alterna a un proceso de planificación institucional, pues si bien existen, no fueron diseñados bajo parámetros del diseño urbano; por el contrario, han sido gestados desde las dinámicas de los ocupantes quienes construyen los espacios colectivos en primera instancia, y luego por uso frecuente promueven una apropiación comunitaria, como se observa con el parque para los niños en "Milagro de Dios", el cual cuentan con un parque construido con material reciclado. 

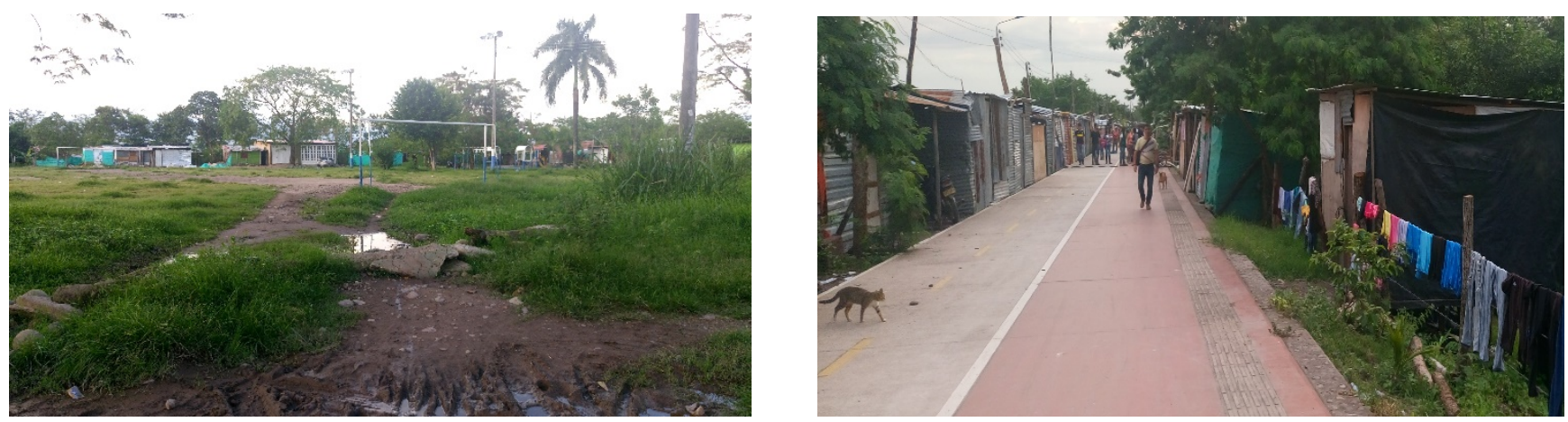

llustración 7. Parque realizado por la comunidad del asentamiento Informal Villa Campestre. Fuente: Elaboración propia llustración 8. Jarillón sobre el cual trasciende la extensión de la vida privada hacia lo público. Se destaca además los tránsitos y la conformación del tejido social en torno a la amenaza por inundación al costado izquierdo de la imagen. Asentamiento Villa Campestre. Fuente: Elaboración propia.

En el cuadro anterior, se muestra el tipo de espacio público que existe en los sectores estudiados, conformado por parques, vías y pequeños equipamientos. Por lo que también se ven importantes las relaciones y construcción del hábitat que se produce en las calles y plazas menores que han sido construidas imitando las costumbres que se desarrollaban previas a la ocupación, por ejemplo en zonas rurales, lo cual también configura la habitar en estos asentamientos. Se plantea así el escenario en que los espacios públicos se ven como lugares creados y forjados a semejanza de costumbres anteriores y que modelan el espacio actual, por lo que los habitantes de los asentamientos se relacionan con los lugares y estos a su vez le dan sentido a las relaciones sociales, creando y reproduciendo los vínculos con el territorio, cargados de subjetividades y costumbres.

De otra parte, las calles y callejones estrechos se relacionan con el espacio público en la medida en que se convierten en sitios de encuentro y tránsito, en los cuales la población juega un rol determinante en el crecimiento de los asentamientos, entonces se tejen allí las formas de relacionar a las personas ocupantes y la extensión de la esfera privada, que en la informalidad tiende a diluirse hacia lo público. Por otra parte, la calidad de vida debe estar en concordancia con la provisión de bienes y servicios, y el desarrollo humano debe propender por las inversiones en capital humano y físico por lo que el mejoramiento del espacio público desde la informalidad es una tarea del día a día, de acuerdo a los representantes de los asentamiento entrevistados, se pretende la adecuación de zonas para el esparcimiento, valga recordar que estos no fueron loteados y tenían clara intención de convertirse en espacio público desde la fundación del asentamiento, comprobando la importancia de este para la consolidación y fortalecimiento de la comunidad y del hábitat a partir de la relación con el entorno.
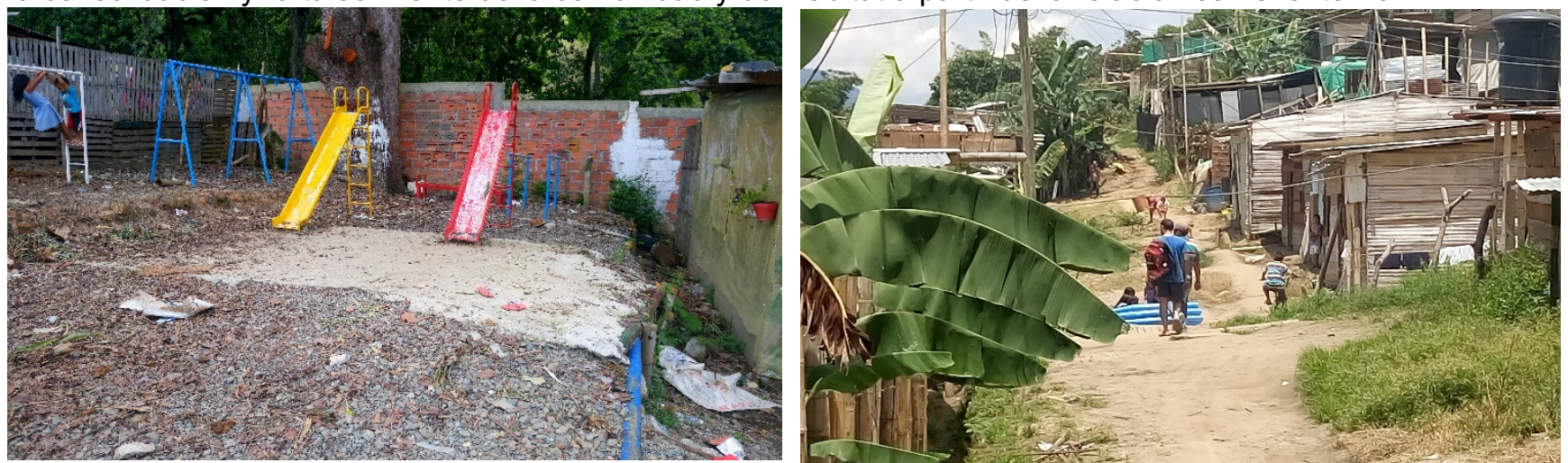

Ilustración 9. Parque y espacio público, construcción del tejido social y apropiación. Fuente: Elaboración propia

Por su parte, no existen canchas deportivas con condiciones para el agrupamiento de las personas; sin embargo, no por ello se debe desconocer que se constituye en una zona central de los asentamientos y se asemeja a plazas o centralidades de la ciudad formal, pues hay encuentros y extensión de las casas hacía estas calles y parques, re-configurando las esferas de lo público y privado que se han idealizado en las típicas ciudades. De 
igual forma, los proceso de la construcción del hábitat desde la informalidad procuran definir espacios donde la relación e integración social en torno a la construcción de un proceso común sea la principal actuación, como se comprobó las calles, patios, jardines y parques son escenarios propicios para el desarrollo de la vida urbana, cultura y costumbres que cada individuo tiene, forjando así la memoria colectiva y la re-significancia cotidiana de los espacios ya que estos son mantenidos, disputados y controlados por los actores de los asentamientos informales.

Lo anterior, es de vital importancia en la construcción y mantenimiento de los asentamientos, pues se genera la apropiación como una manifestación del vínculo social que se teje en torno al territorio, lo que también establece una serie de dinámicas y variables aceptadas en comunidad, así se observa con recelo el consumo de estupefacientes, delitos menores al interior del asentamiento, y es la misma comunidad la que se encarga de controlar estos hechos pues ha existido un proceso de apego y percepción construida desde la base de la informalidad, que intenta generar un contrato social propio con el fin de promover la convivencia.

Ahora bien, es de mencionar que estos asentamientos son constituidos por personas en condición de desplazamiento y vulnerabilidad, distintas procedencias geográficas, ideológicas, etc., lo que para la ciudad formal es un escenario fragmentado y marginado y con unas ínfimas posibilidades de participación, dado que no se cuenta con un título de propiedad y tenencia de los predios; sin embargo, en los casos estudiados se da cuenta que estos son un proceso importante de democratización y participación que permite la creación de vínculos que cohesionan las distintas capas de la sociedad que se encuentran en estos asentamientos, con el fin de realizar intervenciones y mejoras en su proyecto de barrio y construcción de comunidad.

De acuerdo a lo que se mencionó en este apartado, es importante reconocer las identidades propias del lugar, con el fin de que las intervenciones como el mejoramiento barrial no torpedee las decisiones y construcciones anteriormente llevadas a cabo por la comunidad y que están impregnadas de un proceso social. Así, se deben generar espacios que permiten el disfrute de derechos, promuevan la mejora del entorno urbano y con ello la calidad de vida de los habitantes de las zonas hasta ahora informales, promoviendo lo que (Yory, 2015) define como topofilía. En ese sentido, los espacios deben considerar las construcciones culturales y tomar en cuenta la opinión de los "beneficiarios" de los programas, de manera que se tejan visiones abiertas e incluyentes.

\section{Conclusiones}

En este artículo se propendió por realizar una mirada hacia el espacio público desde la informalidad, lo cual sirve de sustento para entender las lógicas sociales desde la autoconstrucción y proponer que debe haber un cambio en las concepciones de políticas y programas de mejoramiento barrial y renovación de espacios, con el fin de que sean tenidas en cuentas las subjetividades de la comunidad y así evitar la perpetuación de prácticas segregadoras y el mantenimiento de roles que perjudican las relaciones sociales y con ellas al territorio. Valga decir, que en ocasiones como lo señalan (Pérez y Castellano, 2013) cuando no se genera una cohesión con la comunidad, la respuesta es negativa y se abandonan los lugares, pues no se consideran espacios de la comunidad, entonces se degradan y terminan por ser olvidados.

Igualmente, el papel de los urbanizadores piratas es el de vender la mayor cantidad de predios y lotear sin tener claro un modelo urbanístico que comprenda, entre otras cosas, el espacio público relacionado con las calles, parques, etc. Por lo que el espacio público en la informalidad tiende a perder el carácter que en el urbanismo clásico se le ha dado como espacios de encuentro, caracterizados por ser amplios y que respetan algunos preceptos. Sin embargo, y como se dio cuenta, desde la informalidad se adecuan espacios y se les dota de significados para su uso, por lo que sí existen estos espacios públicos pero alternos al urbanismo y diseño, por ejemplo las vías internas se convierten en hitos que son defendidos y transitados.

Como se advirtió, algunos programas de mejoramiento y de recuperación del espacio público carecen de una mirada interpretativa que permiten tener en cuenta las subjetividades, los intereses, así como los factores contextuales con los que fueron construidos y mantenidos por la comunidad, relacionadas con el hábitat, por lo que el estudio entre otras, debería permitir adoptar una perspectiva amplia e integral sobre estas áreas, a partir de reconocimiento de elementos: cognitivos, discursivos, argumentativos, retóricos y narrativos, que entienden la construcción del espacio en donde se desarrolla la informalidad como resultado de luchas entre actores con relaciones de poder, para el caso de las ciudades "formales" y la "otra" ciudad. 
Como se dijo anteriormente, la construcción del hábitat a partir de un proceso de autogestión se debe a varios factores y no se da de igual manera en todos los territorios, por cuanto una política homogeneizadora de reasentamiento, no responde a las lógicas propias. Por cuanto, es necesario ubicar dichas diferencias y similitudes sin perder de vista el hábitat como constructo, así como el acceso al espacio público implican relaciones de poder y dominación por parte de actores sobre el territorio, como lo señala (Lindon, 2007)esto puede ser expuesto mediante la identificación de las representaciones sociales, las cuales visibilizan consensos que de ser abordados de manera amplia posibilitarían la tarea de construir mejores políticas de mejoramiento que no caigan en la reproducción de imaginarios discriminatorios y excluyentes, pero que responden a lógicas económicas y mundializadoras, en contraposición se deben realizar procesos consensuados y que respeten las concepciones por parte de los actores que han formado su hábitat y relaciones.

Finalmente, la creciente apertura de la escena pública genera en la sociedad un mayor protagonismo, por lo que las políticas y programas tendientes a la reconfiguración de la ciudad, debe aumentar los mecanismos e instancias donde se responsabilicen a los actores, con el fin de generar una intervención más directa en la construcción de las políticas. El desafío va en poner en marcha la relación entre la formulación, implementación y evaluación del proceso de políticas, conociendo hacia dónde va y, evaluar si era a donde realmente se llegó, o por el contrario cuanto se apartó de las metas, logrando identificar cómo la población se hace decisiva cuando participa en el proceso de implementación de políticas de mejoramiento barrial, construidas desde sus vivencias y con la perspectiva del derecho a la ciudad y al espacio público.

\section{Bibliografía}

Aguliar, J. y. (2013). Localización de los pobres: barrio, frente a las características del hogar: el caso de Bogotá. GDN Working paper(73).

Augé, M. (1996). Espacios del anonimato. Una antropología de la sobre modernidad. Buenos Aires:Paidós. . Barcelona: Editorial Gedisa.

Delgado, O. (2003). Debates sobre el espacio en la geografía contemporánea. . Bogotá : Universidad Nacional de Colombia. .

Duque, I. (2015). Inclusione ed equita territoriale nell'agenda di planificazione urbana a Medellín (Colombia). Bolletino della Societa Geografica Italiana Roma, VIII, 109-121.

Echeverría, M. C. (2009). Hábitat: Concepto, campo y trama de vida . En M. C. Echeverría, ¿Qué es el hábitat?: La preguntas por el hábitat. Medellín : Facultad de arquitectura. Universidad Nacional de Colombia. Sede Medellín.

Fischer, F. (2003). Reframing public policy: discursive politics and deliberative practices. . Oxford : Oxford University Press. .

Giraldo, F. (2004). Hábitat y desarrollo humano. washington: Cuaderno PNUD, UN Hábitat: Investigaciones sobre desarrollo humano.

Jacobs, J. (1961 ). Muerte y Vida de las grandes ciudades . Madrid : Traducido por: Abad. A y Useros A.(2011) Colección entrelineas .

Leff, E. (2002). Saber ambiental, sutentabilidad, racionalidad, complejidad, poder. México: Editorial Siglo XXI.

Lindon, A. (2007). La ciudad y la vida urbana a través de los imaginarios urbano. Revista Eure, XXXIII(99), 7-16.

Majone, G. (1997). Evidencia, argumentación y persuación en la formulación de políticas. . México D.F: Fondo de Cultura Económica.

Ortíz, E. (2004). Notas sobre la producción social de vivienda. Elementos básicos para su coneptualización en impulso . México D.F: Coalición internacional del Hábitat .

Páramo, P. y. (2009). La experiencia urbana en el espacio público de Bogotá en el siglo XX: Una mirada desde las prácticas sociales. Bogotá: Universidad Pedagógica Nacional.

Pérez, T. y. (2013). Creación del espacio público en asentamientos informales: Nuevos desafios urbanos. Bitacora, 41(95), 95-104.

Raffestin, C. (1993). Por una geografía del poder. Sao Paulo: Atica.

Sabaté, A. (1995). Teoría y práctica de la geografía del género. En J. M. Mujeres, espacio y sociedad. Hacia una geografía del género. En Sabaté Martínez. Madrid: Editorial Síntesis.

Sabatini, F. y. (2012). La segregación, los guetos y loa integración social urbana: mitos y claves. Revista Eure, 526. 
Sepúlveda, R. D. (1992). Enfoque sistémico y lugar: Una perspectiva para el estudio de hábitat residenciales urbanos. Santiago de Chile : Instituto de la Vivienda. Universidad de Chile.

Soja, E. (2009). The City and spatial justice. N. 1. Septiembre de 2009. Conference spatial justice, Nanterre, Paris. March 12-14 (2008)(1).

Velásquez, R. (2009). Hacia una nueva definición del concepto "política pública". Desafíos (20), 149-187. .

Yory, C. (2015). La construcción social del Hábitat: como estrategia de integración social, sustentabilidad urbana y seguridad ciudadana. Bogotá : Universidad Piloto de Colombia. 